\title{
Global Regularity in Fractional Order Sobolev Spaces for the p-Laplace Equation on Polyhedral Domains
}

\author{
C. Ebmeyer, WB. Liu and M. Steinhauer
}

\begin{abstract}
The $p$-Laplace equation is considered for $p>2$ on a $n$-dimensional convex polyhedral domain under a Dirichlet boundary value condition. Global regularity of weak solutions in weighted Sobolev spaces and in fractional order Nikolskij and Sobolev spaces are proven.
\end{abstract}

Keywords: p-Laplace equation, global regularity, fractional order Nikolskij spaces MSC 2000: Primary 35J55, 35J65, secondary 35J25

\section{Introduction}

Let $\Omega \subset \mathbb{R}^{n}$ be a convex polyhedral domain and $p>2$. We will be concerned with the Dirichlet problem for the elliptic $p$-Laplace equation

$$
\begin{aligned}
-\operatorname{div}\left(|\nabla u(x)|^{p-2} \nabla u(x)\right) & =f(x) & & \text { in } \Omega \\
u(x) & =0 & & \text { on } \partial \Omega,
\end{aligned}
$$

where $u: \Omega \rightarrow \mathbb{R}, f \in W^{-1, p^{\prime}}(\Omega)$, and $p^{\prime}=\frac{p}{p-1}$. Our aim is to prove global regularity results for weak solutions $u$ under suitable assumptions on the righthand side $f$.

Problems with $p$-structure arise in many physical contexts, such as in plasticity theory, bimaterial problems in elastic-plastic mechanics, and nonNewtonian fluids; see, e.g., $[1,8,11,13]$ and the references given therein. The $p$-Laplace operator may be seen as the prototype of problems with $p$-structure,

C. Ebmeyer and M. Steinhauer: Mathematisches Seminar, Universität Bonn, Nussallee 15, D-53115 Bonn, Germany; carsten.ebmeyer@uni-bonn.de, steinhauer@ uni-bonn.de

WB. Liu: CBS \& IMS, University of Kent, Canterbury, CT2 7NF, England;

W.B.Liu@kent.ac.uk

ISSN 0232-2064 / \$2.50 C Heldermann Verlag Berlin 
as the Laplace operator is for usual elliptic problems. Despite this fact, global regularity results in Sobolev spaces are in general not available. For the Laplace equation (i.e., $p=2$ ) it is well-known that weak solutions of the homogeneous Dirichlet problem on bounded domains are $W^{2,2}(\Omega)$-functions, if the right-hand side $f$ and the boundary of the domain are suitably smooth. In this paper we generalize this result to the $p$-Laplacian for $p>2$. We will show that each weak solution $u$ of the $p$-Laplace equation satisfies

$$
u \in \mathcal{N}^{1+\frac{2}{p}, p}(\Omega) \quad \text { for } p>2,
$$

where $\mathcal{N}^{s, p}$ is a Nikolskij space. This implies that $u \in W^{1+s, p}(\Omega)$ for all $s<\frac{2}{p}$. Moreover, we will prove

$$
\int_{\Omega}|\nabla u|^{p-2}\left|\nabla^{2} u\right|^{2} d x<\infty
$$

This result is known on interior domains $\Omega_{0} \subset \subset \Omega$ under some strong regularity assumptions on $f$ such as $f=0$, or for the nondegenerate case (that is, $-\operatorname{div}(1+$ $\left.\left.|\nabla u|^{p-2}\right) \nabla u=f\right)$; see $[3,9,14,15,17]$. Further, for $p<2$ and smooth domains the regularity result (3) was shown in [12].

The main ingredient in our proof is a refinement of the difference quotient technique, as developed in $[4,6]$ in order to investigate the regularity of weak solutions of nonlinear problems. Our method of proof can be applied to a wide range of problems with $p$-structure, such as convex domains with piecewise smooth boundaries, nonhomogeneous boundary value conditions, and systems of equations. However, for simplicity, we restrict ourselves to convex polyhedrons in $\mathbb{R}^{n}$ and homogeneous Dirichlet boundary value conditions. We require only minimal assumptions on the regularity of the right-hand side $f$. In particular, we do not assume regularity in weighted spaces.

Regularity results of this type have an interest in their own but they are very useful for numerical purposes, as well. In [7] error bounds for finite element approximations of problems with $p$-structure are proven. It is shown that the regularity results (2) and (3) are sufficient for deriving the optimal rates of convergence. We refer to [7] for a detailed discussion related to this subject.

This paper is organized as follows. In Section 2 the main results are given. In Section 3 we treat the local situation near a flat boundary portion and prove the basic estimates by applying a difference quotient method. In Section 4 we investigate the local situation near corner points and nonsmooth portions of the boundary. Section 5 contains the proofs of the main results.

\section{Main results and notations}

In this paper we are concerned with convex polyhedral domains. More precisely, we consider convex domains satisfying the following assumptions: 
i) $\Omega \subset \mathbb{R}^{n}$ is a bounded, convex, open set in $\mathbb{R}^{n}$ for $n \geq 2$;

ii) $\partial \Omega=\bigcup_{1 \leq k \leq M} \overline{\Gamma_{k}}$, where $\Gamma_{k}$ for $k=1, \ldots, M$ are relative open subsets of a hyperplane, and each $\Gamma_{k}$ is a $(n-1)$-dimensional convex polyhedral domain;

iii) $\Gamma_{i} \cap \Gamma_{k}=\emptyset$ for $i \neq k$;

iv) $\partial \Gamma_{k_{1}} \cap \ldots \cap \partial \Gamma_{k_{j}}=\emptyset$, if $j>n$ and $k_{1}<\ldots<k_{j}$.

Condition iv) means that the closures of at most $n$ "faces" of the boundary have a nonempty intersection. Typical examples of such domains are, for instance, cubes or tetrahedrons in three dimensions, and classical convex polygonal domains in two dimensions.

By $W^{s, p}(\Omega)$ we denote the usual Sobolev spaces. In the case that $s>0$ is no integer, a function $f: \Omega \rightarrow \mathbb{R}$ belongs to $W^{s, p}(\Omega)$, if the norm

$$
\|f\|_{W^{s, p}(\Omega)}=\left(\|f\|_{W^{m, p}(\Omega)}^{p}+\sum_{|\alpha|=m} \int_{\Omega} \int_{\Omega} \frac{\left|\partial^{\alpha} f(x)-\partial^{\alpha} f(y)\right|^{p}}{|x-y|^{n+\sigma p}} d x d y\right)^{\frac{1}{p}}
$$

is finite, where $s=m+\sigma, m \geq 0$ is an integer, $0<\sigma<1$, and $1 \leq p<\infty$. Further, we use the Nikolskij spaces $\mathcal{N}^{s, p}(\Omega)$; cf. [10]. Let $\Omega^{\delta}=\{x \in \Omega$ : $\operatorname{dist}(x, \partial \Omega) \geq \delta\}$ and $z \in \mathbb{R}^{n}$. The space $\mathcal{N}^{s, p}(\Omega)$ consists of all functions $f: \Omega \rightarrow \mathbb{R}$ for which the norm

$$
\|f\|_{\mathcal{N}^{s, p}(\Omega)}=\left(\|f\|_{L^{p}(\Omega)}^{p}+|f|_{\mathcal{N}^{s, p}(\Omega)}^{p}\right)^{\frac{1}{p}}
$$

is finite, where as before $s=m+\sigma, m \in \mathbb{N}_{0}, 0<\sigma<1$, and

$$
|f|_{\mathcal{N}^{s, p}(\Omega)}^{p}=\sum_{|\alpha|=m} \sup _{0<|z|<\delta} \int_{\Omega^{\delta}} \frac{\left|\partial^{\alpha} f(x+z)-\partial^{\alpha} f(x)\right|^{p}}{|z|^{\sigma p}} d x .
$$

The spaces $\mathcal{N}^{s, p}(\Omega)$ are larger than the corresponding Sobolev spaces $W^{s, p}(\Omega)$; but for $\varepsilon>0$ we have the imbeddings [10, section 8.2.5], [16, section 6.2]

$$
\mathcal{N}^{s+\varepsilon, p}(\Omega) \hookrightarrow W^{s, p}(\Omega) \hookrightarrow \mathcal{N}^{s, p}(\Omega)
$$

Let us introduce the notations $\partial_{i}:=\frac{\partial}{\partial x_{i}},|\nabla u|^{2}:=\sum_{i=1}^{n}\left(\partial_{i} u\right)^{2}:=\partial_{i} u \partial_{i} u$, and $\left|\nabla^{2} u\right|^{2}:=\sum_{i, j=1}^{n}\left(\partial_{i} \partial_{j} u\right)^{2}:=\partial_{i} \partial_{j} u \partial_{i} \partial_{j} u$ by employing Einstein's summation convention saying that one has to sum over an index that occurs twice.

We call a function $u \in W_{0}^{1, p}(\Omega)$ a weak solution of (1), if

$$
\int_{\Omega}|\nabla u|^{p-2} \partial_{i} u \partial_{i} \phi d x=\int_{\Omega} f \phi d x \quad \text { for all } \phi \in W_{0}^{1, p}(\Omega)
$$


It is well-known that for $1<p<+\infty$ and $f \in W^{-1, p^{\prime}}(\Omega)$ there exists a unique weak solution of (1), because (5) is the weak formulation of the Euler-Lagrange equation of the variational integral

$$
\mathcal{D}_{p}(u ; \Omega):=\frac{1}{p} \int_{\Omega}|\nabla u|^{p} d x-\int_{\Omega} f u d x
$$

that is strictly convex. So, in fact the weak solution of (1) realizes the unique minimum of $\mathcal{D}_{p}(u ; \Omega)$ over $W_{0}^{1, p}(\Omega)$.

Now we state our main results. The first theorem is concerned with the regularity of weak solutions in fractional order Nikolskij spaces.

Theorem 2.1. Let $p>2$ and $f \in W^{\frac{p-2}{p}, p^{\prime}}(\Omega)$. Then the weak solution $u$ of $(5)$ satisfies

$$
u \in \mathcal{N}^{1+\frac{2}{p}, p}(\Omega) .
$$

Remark. i) Theorem 2.1 implies corresponding regularity of $u$ in fractional order Sobolev spaces. Utilizing the imbedding theorem of Nikolskij spaces into Sobolev spaces (4) we get

$$
u \in W^{1+\frac{2}{p}-\varepsilon, p}(\Omega) \quad \text { for all } \varepsilon>0 .
$$

In this statement the $\varepsilon$ comes in only by using the imbedding theorem of Nikolskij spaces into Sobolev spaces (4). This result may be seen as a generalization of the well-known fact that a weak solution of the Laplace equation (i.e., $p=2$ ) is a $W^{2,2}(\Omega)$-function (and therefore certainly also a $\mathcal{N}^{2,2}(\Omega)$-function (c. f. (4) with $s=p=2$.)).

ii) In order to prove our results we test the equation by second order difference quotients of $u$. In [5] difference quotients of first order were used in order to investigate the regularity for the $p$-Laplace equation on domains with corners, i.e., on nonconvex polyhedrons. Instead of (7) it was shown that $u \in W^{1+\frac{1}{p}-\varepsilon, p}(\Omega)$ for all $\varepsilon>0$. This generalizes the fact that weak solutions of the Laplace equation on nonconvex polyhedrons are $W^{\frac{3}{2}, 2}(\Omega)$-functions.

The next theorem provides regularity of the second derivatives in a weighted Sobolev space.

Theorem 2.2. Let $p>2$ and $f \in W^{\frac{p-2}{p}, p^{\prime}}(\Omega)$. Then the weak solution $u$ of $(5)$ satisfies

$$
\int_{\Omega}|\nabla u|^{p-2}\left|\nabla^{2} u\right|^{2} d x \leq c
$$

where the constant $c$ depends only on the data, i.e., $c=c(n, p, \Omega, f)$. 
Remark. i) Let us discuss the sharpness of our results. The function $u=|x|^{\alpha+\varepsilon}$ with $\alpha=\frac{p+2-n}{p}$ solves $-\Delta_{p} u=f$, where $f=c|x|^{(\alpha+\varepsilon)(p-1)-p}$. If $\varepsilon>0$ then $f \in$ $W^{\frac{p-2}{p}, p^{\prime}}(\Omega)$ and $u$ satisfies (6) and (8). However, if $\varepsilon=0$, then $f \notin W^{\frac{p-2}{p}, p^{\prime}}(\Omega)$, $|\nabla u|^{p-2}\left|\nabla^{2} u\right|^{2} \notin L^{1}(\Omega)$, and $u \notin \mathcal{N}^{s, p}(\Omega)$ for all $s>1+\frac{2}{p}$.

ii) Theorem 2.2 implies that

$$
|\nabla u|^{\frac{p-2}{2}} \nabla u \in W^{1,2}\left(\Omega ; \mathbb{R}^{n}\right)
$$

iii) Theorem 2.1 and the imbedding theorem of Nikolskij spaces into Nikolskij spaces (with different differentiability and integrability index) provides $[10$, Section 8.2.9 f.], [16, Section 6.3]

$$
u \in \mathcal{N}^{1, \frac{n p}{n-2}}(\Omega) \quad \text { for } n \geq 3, \quad u \in \mathcal{N}^{1, \infty}(\Omega) \quad \text { for } n=2 .
$$

iv) Utilizing (9) and well-known imbedding theorems we obtain higher integrability of $\nabla u$ and Hölder continuity of $u$ (for suitable values of the parameter $p)$. In fact, for $n \geq 3$ we get

$$
\nabla u \in L^{\frac{n p}{n-2}}\left(\Omega ; \mathbb{R}^{n}\right), \quad u \in C^{0,1+\frac{2}{p}-\frac{n}{p}}(\bar{\Omega}) \quad \text { if } p>\max (2, n-2) .
$$

In the case of $n=2$ it follows that

$$
\nabla u \in L^{q}\left(\Omega ; \mathbb{R}^{n}\right) \quad \forall q<+\infty, \quad u \in C^{0, \alpha}(\bar{\Omega}) \quad \forall \alpha \in(0,1)
$$

Remark. Possible generalizations of our results resp. method of proof concern the following topics:

i) Bounded domains $\Omega$ with piecewise $W^{2, \infty} \equiv C^{1,1}$ boundary $\partial \Omega$ and convex angles.

ii) More general equations with $p$-structure under suitable growth- and monotonicity assumptions on the coefficients.

iii) The $p$-Laplace system

$$
-\partial_{j}\left(|\nabla u(x)|^{p-2} \partial_{j} u^{i}(x)\right)=f^{i}(x) \quad \text { in } \Omega, \quad u^{i}(x)=0 \quad \text { on } \partial \Omega,
$$

for all $1 \leq i \leq N$, where $u: \Omega \rightarrow \mathbb{R}^{N}$ and $N>1$.

iv) More general boundary value conditions such as inhomogeneous Dirichlet or Neumann boundary value conditions.

Let us remark that all these generalizations are straightforward. Indeed, we are able to treat situations which arise after a $W^{2, \infty}$-mapping of a polyhedron onto a domain with a piecewise smooth boundary; cf. [4, 6]. Moreover, let us note that there is no difference in treating systems or equations. The proof is the same just with indices inserted. 


\section{Local regularity up to a flat boundary portion}

Let $R>0, P \in \partial \Omega$, and $B_{R}(P)=\left\{x \in \mathbb{R}^{n}:|x-P|<R\right\}$. In this section we investigate the regularity of $u$ in $\Omega \cap B_{4 R}(P)$ under the assumption that

$$
\partial \Omega \cap B_{4 R}(P)=\Gamma_{k_{0}} \cap B_{4 R}(P)
$$

for some $k_{0} \in\{1, \ldots, M\}$. That is, we assume that $\partial \Omega \cap B_{4 R}(P)$ is contained in a hyperplane. First, we investigate the regularity of $u$ in a direction tangential to $\Gamma_{k_{0}}$, then in a direction normal to $\Gamma_{k_{0}}$; see the Lemmas 3.3 and 3.4 below. The aim of this section is to show that some expressions containing a difference quotient of $\nabla u$ stay uniformly bounded in $L^{2}$. For convenience, we will assume that $f \in W^{1, p^{\prime}}(\Omega)$. The weaker assumption $f \in W^{\frac{p-2}{p}, p^{\prime}}(\Omega)$ will be treated in Section 5 .

Our method of proof is a difference quotient technique. Let us introduce some notations. Let $h>0$, and let $e \in \mathbb{R}^{n}$ be a unit vector, i.e., $|e|=1$. We set

$$
T_{e}^{h} u(x):=u(x+h e), \quad T_{e}^{-h} u(x):=u(x-h e)
$$

as well as

$$
\begin{aligned}
D_{e}^{h} u(x) & :=\frac{T_{e}^{h} u(x)-u(x)}{h}=\frac{u(x+h e)-u(x)}{h} \\
D_{e}^{-h} u(x) & :=\frac{u(x)-T_{e}^{-h} u(x)}{h}=\frac{u(x)-u(x-h e)}{h} .
\end{aligned}
$$

That is, $D_{e}^{h}$ is the forward difference quotient of $u$ in direction $e$ and $D_{e}^{-h}$ the backward difference quotient. To shorten our writing we use the abbreviations $B_{R}=B_{R}(P), \Omega_{R}=\Omega \cap B_{R}(P)$. Further, $\langle\cdot, \cdot\rangle$ denotes the Euclidean scalar product in $\mathbb{R}^{n}$ and $C$ will denote a generic constant that will be allowed to vary from equation to equation or from inequality to inequality.

Remark. i) If the function $u=u(x)$ is defined in some open set $\Omega \subset \mathbb{R}^{n}$, then $D_{e}^{h} u$ and $D_{e}^{-h} u$ are defined in the sets $\{x \in \Omega: x \pm h e \in \Omega\}$ and therefore in

$$
\Omega_{0}:=\{x \in \Omega: \operatorname{dist}(x, \partial \Omega)>h\} .
$$

ii) If $u \in W^{1, p}(\Omega)$, then $D_{e}^{ \pm h} u \in W^{1, p}\left(\Omega_{0}\right)$, and we have $\partial_{i} D_{e}^{ \pm h} u=D_{e}^{ \pm h} \partial_{i} u$. iii) If at least one of the functions $u, v$ has a support contained in $\Omega_{0}$, then the discrete integration by parts formula holds, i.e.,

$$
\int_{\Omega} u D_{e}^{ \pm h} v d x=-\int_{\Omega} v D_{e}^{\mp h} u d x .
$$


iv) We have the following discrete Leibniz product rules for difference quotients of first order:

$$
\begin{aligned}
D_{e}^{h}(u v)(x) & =T_{e}^{h} u(x) D_{e}^{h} v(x)+v(x) D_{e}^{h} u(x), \\
D_{e}^{-h}(u v)(x) & =T_{e}^{-h} u(x) D_{e}^{-h} v(x)+v(x) D_{e}^{-h} u(x),
\end{aligned}
$$

and for difference quotients of second order:

$$
\begin{aligned}
D_{e}^{h} D_{e}^{h}(u v)(x)= & T_{e}^{2 h} u(x) D_{e}^{h} D_{e}^{h} v(x)+D_{e}^{h} v(x) T_{e}^{h} D_{e}^{h} u(x) \\
& +D_{e}^{h} u(x) D_{e}^{h} v(x)+T_{e}^{h} v(x) D_{e}^{h} D_{e}^{h} u(x) \\
D_{e}^{-h} D_{e}^{-h}(u v)(x)= & u(x) D_{e}^{-h} D_{e}^{-h} v(x)+T_{e}^{-h} D_{e}^{-h} v(x) D_{e}^{-h} u(x) \\
& +T_{e}^{-h} D_{e}^{-h} u(x) T_{e}^{-h} D_{e}^{-h} v(x)+T_{e}^{-h} v(x) D_{e}^{-h} D_{e}^{-h} u(x) \\
D_{e}^{h} D_{e}^{-h}(u v)(x)= & u(x) D_{e}^{h} D_{e}^{-h} v(x)+D_{e}^{-h} u(x) D_{e}^{-h} v(x) \\
& +T_{e}^{h} v(x) D_{e}^{h} D_{e}^{-h} u(x)+D_{e}^{-h} u(x) D_{e}^{h} v(x) \\
= & D_{e}^{-h} D_{e}^{h}(u v)(x) .
\end{aligned}
$$

v) Let $B_{3 r}\left(x_{0}\right)$ be a ball and $v \in W^{1, p}\left(B_{3 r}\left(x_{0}\right)\right)$. Then there exists a constant $K>0$ such that

$$
\sup _{0<h<r} \int_{B_{2 r}\left(x_{0}\right)}\left|D_{e}^{ \pm h} v(x)\right|^{p} d x \leq K \int_{B_{3 r}\left(x_{0}\right)}|\langle\nabla v, e\rangle|^{p} d x \leq K \int_{B_{3 r}\left(x_{0}\right)}|\nabla v|^{p} d x .
$$

vi) Let $v \in L^{p}(\Omega), 1<p<+\infty$, and suppose there exist constants $K>0$ and $h_{0}>0$ such that

$$
\sup _{e \in \mathbb{R}^{n},|e|=1} \sup _{0<h<h_{0}}\left\|D_{e}^{ \pm h} v\right\|_{L^{p}\left(\Omega_{0}\right)} \leq K
$$

Then $\nabla v \in L^{p}(\Omega)$ and $\|\nabla v\|_{L^{p}(\Omega)} \leq K$. Furthermore, $D_{e}^{ \pm h} v \rightarrow\langle\nabla v, e\rangle$ in $L_{l o c}^{p}(\Omega)$ as $h \downarrow 0$.

For a discussion and proofs of these statements we refer to [9, pp. $271 \mathrm{ff}$.]. Now we prove two auxiliary lemmas. We start with a technical result.

Lemma 3.1. Let $\alpha \geq 0$ and $\beta>0$. There exist two constants $c_{1}(\alpha, \beta)$ and $c_{2}(\alpha, \beta)$ such that

$$
c_{1}\left(|a|^{2}+|b|^{2}\right)^{\frac{\beta}{2}} \leq \int_{0}^{1}(1-t)^{\alpha}|t a+(1-t) b|^{\beta} d t \leq c_{2}\left(|a|^{2}+|b|^{2}\right)^{\frac{\beta}{2}}
$$

for all vectors $a, b \in \mathbb{R}^{n}$. 
Proof. Let $Z(t)=|t a+(1-t) b|$. The estimate from above is obvious:

$$
\begin{aligned}
\int_{0}^{1}(1-t)^{\alpha} Z(t)^{\beta} d t & \leq \int_{0}^{1} Z(t)^{\beta} d t \\
& =\int_{0}^{1}\left(|t a+(1-t) b|^{2}\right)^{\frac{\beta}{2}} d t \\
& \leq \int_{0}^{1}\left(2 t^{2}|a|^{2}+2(1-t)^{2}|b|^{2}\right)^{\frac{\beta}{2}} d t \\
& \leq 2^{\frac{\beta}{2}}\left(|a|^{2}+|b|^{2}\right)^{\frac{\beta}{2}} .
\end{aligned}
$$

So we can take $c_{2}=2^{\frac{\beta}{2}}$. For the estimate from below we first suppose that $|a| \geq|b|$. Then we have for $t \geq \frac{2}{3} Z(t) \geq t|a|-(1-t)|b| \geq(2 t-1)|b| \geq \frac{1}{3}|b|$ and also $Z(t) \geq t|a|-(1-t)|b| \geq(2 t-1)|a| \geq \frac{1}{3}|a|$. Both together yield

$$
Z(t) \geq \frac{1}{6}(|a|+|b|) \quad \text { for } t \geq \frac{2}{3} \text {. }
$$

In the same manner we obtain for $|b| \geq|a|$ and $t \leq \frac{1}{3}$ the same inequality. Therefore,

$$
\begin{aligned}
& \int_{0}^{1}(1-t)^{\alpha} Z(t)^{\beta} d t \geq \int_{\frac{2}{3}}^{1}(1-t)^{\alpha} Z(t)^{\beta} d t \geq \frac{1}{(\alpha+1) 3^{\alpha+1} 6^{\beta}}(|a|+|b|)^{\beta} \\
& \int_{0}^{1}(1-t)^{\alpha} Z(t)^{\beta} d t \geq \int_{0}^{\frac{1}{3}}(1-t)^{\alpha} Z(t)^{\beta} d t \geq \frac{3^{\alpha+1}-2^{\alpha+1}}{(\alpha+1) 3^{\alpha+1} 6^{\beta}}(|a|+|b|)^{\beta} .
\end{aligned}
$$

Taking $\left(|a|^{2}+|b|^{2}\right)^{\frac{\beta}{2}} \leq(|a|+|b|)^{\beta}$ into account the assertion follows with

$$
c_{1}=\frac{\min \left(1,3^{\alpha+1}-2^{\alpha+1}\right)}{(\alpha+1) 3^{\alpha+1} 6^{\beta}} .
$$

The following lemma is a convexity-like inequality and provides a basic estimate of our proof. (In the sequel we silently assume that all functions are extended in a suitable manner onto a $h$-neighbourhood of $\Omega$.)

Lemma 3.2. Let $0<h<\frac{R}{2}, P \in \bar{\Omega}$, and $\eta \in W^{2, \infty}\left(\mathbb{R}^{n}\right)$ be a cut-off function satisfying $\eta \equiv 1$ in $B_{R}(P)$, supp $\eta=\overline{B_{2 R}}$, and $0 \leq \eta \leq 1$ in $\mathbb{R}^{n}$. For every unit vector $e \in \mathbb{R}^{n}$ and every $v \in W^{1, p}(\Omega)$ there holds

$$
\begin{aligned}
c_{1} & \int_{\Omega_{3 R}} \eta^{2}\left(\left|T_{e}^{h} \nabla v\right|^{2}+|\nabla v|^{2}\right)^{\frac{p-2}{2}}\left|D_{e}^{h} \nabla v\right|^{2} d x \\
+c_{1} & \int_{\Omega_{3 R}} \eta^{2}\left(\left|T_{e}^{-h} \nabla v\right|^{2}+|\nabla v|^{2}\right)^{\frac{p-2}{2}}\left|D_{e}^{-h} \nabla v\right|^{2} d x \\
& \leq \frac{1}{p} \int_{\Omega_{3 R}} \eta^{2} D_{e}^{h} D_{e}^{-h}|\nabla v|^{p} d x-\int_{\Omega_{3 R}} \eta^{2}|\nabla v|^{p-2}\left\langle\nabla v, D_{e}^{h} D_{e}^{-h} \nabla v\right\rangle d x
\end{aligned}
$$

where the constant $c_{1}$ depends only on $p$ and is determined as in Lemma 3.1. 
Proof. Let $0<h<\frac{R}{2}, z \in \mathbb{R}^{n}$, and $\partial_{i}=\frac{\partial}{\partial z_{i}}$. Defining the functions $a(z)=\frac{1}{p}|z|^{p}$ and

$$
\begin{aligned}
a_{i}(z) & =\partial_{i} a(z)=|z|^{p-2} z_{i} \\
a_{i j}(z) & =\partial_{j} a_{i}(z)=(p-2)|z|^{p-4} z_{i} z_{j}+|z|^{p-2} \delta_{i j},
\end{aligned}
$$

the Taylor expansion of $a(z)$ yields

$$
\begin{aligned}
a\left(z^{\prime}\right)-a(z) & -\left(z^{\prime}-z\right)_{i} a_{i}(z) \\
& =\left(z^{\prime}-z\right)_{i}\left(z^{\prime}-z\right)_{j} \int_{0}^{1}(1-s) a_{i j}\left(s z^{\prime}+(1-s) z\right) d s .
\end{aligned}
$$

Let us put $z^{\prime}=T_{e}^{h} \nabla v$ and $z=\nabla v$. Due to the fact that

$$
a_{i j}(z) \xi_{i} \xi_{j}=|z|^{p-2}|\xi|^{2}+(p-2)|z|^{p-4}\langle z, \xi\rangle^{2} \geq|\xi|^{2}|z|^{p-2}
$$

for all $\xi \in \mathbb{R}^{n}$ and $z \in \mathbb{R}^{n}$ we get

$$
\begin{aligned}
\frac{1}{p}\left|T_{e}^{h} \nabla v\right|^{p}-\frac{1}{p}|\nabla v|^{p}-\left\langle|\nabla v|^{p-2} \nabla v, \nabla T_{e}^{h} v-\nabla v\right\rangle \\
=\left|T_{e}^{h} \nabla v-\nabla v\right|^{2} \int_{0}^{1}(1-s)\left|\mu_{e}^{h}(s, \nabla v)\right|^{p-2} d s \\
\quad+(p-2) \int_{0}^{1}(1-s)\left|\mu_{e}^{h}(s, \nabla v)\right|^{p-4}\left\langle\mu_{e}^{h}(s, \nabla v), \nabla T_{e}^{h} v-\nabla v\right\rangle^{2} d s \\
\geq\left|T_{e}^{h} \nabla v-\nabla v\right|^{2} \int_{0}^{1}(1-s)\left|\mu_{e}^{h}(s, \nabla v)\right|^{p-2} d s
\end{aligned}
$$

where $\mu_{e}^{h}(s, \nabla v)=s T_{e}^{h} \nabla v+(1-s) \nabla v$. Multiplying by $\eta^{2}$, integrating over $\Omega$, and dividing by $h$ it follows that

$$
\begin{aligned}
\int_{\Omega_{3 R}} \eta^{2} D_{e}^{h} & \frac{|\nabla v|^{p}}{p} d x-\int_{\Omega_{3 R}} \eta^{2}|\nabla v|^{p-2}\left\langle\nabla v, D_{e}^{h} \nabla v\right\rangle d x \\
& \geq h \int_{\Omega_{3 R}} \eta^{2}\left|D_{e}^{h} \nabla v\right|^{2} \int_{0}^{1}(1-s)\left|\mu_{e}^{h}(s, \nabla v)\right|^{p-2} d s d x .
\end{aligned}
$$

Similarly, putting $z^{\prime}=T_{e}^{-h} \nabla v$ and $z=\nabla v$ in equation (12) we find

$$
\begin{aligned}
-\int_{\Omega_{3 R}} \eta^{2} & D_{e}^{-h} \frac{|\nabla v|^{p}}{p} d x+\int_{\Omega_{3 R}} \eta^{2}|\nabla v|^{p-2}\left\langle\nabla v, D_{e}^{-h} \nabla v\right\rangle d x \\
= & h \int_{\Omega_{3 R}} \eta^{2}\left|D_{e}^{-h} \nabla v\right|^{2} \int_{0}^{1}(1-s)\left|\mu_{e}^{-h}(s, \nabla v)\right|^{p-2} d s d x+h(p-2) \\
& \times \int_{\Omega_{3 R}} \eta^{2} \int_{0}^{1}(1-s)\left|\mu_{e}^{-h}(s, \nabla v)\right|^{p-4}\left\langle\mu_{e}^{-h}(s, \nabla v), D_{e}^{-h} \nabla v\right\rangle^{2} d s d x \\
\geq & h \int_{\Omega_{3 R}} \eta^{2}\left|D_{e}^{-h} \nabla v\right|^{2} \int_{0}^{1}(1-s)\left|\mu_{e}^{-h}(s, \nabla v)\right|^{p-2} d s d x
\end{aligned}
$$


where $\mu_{e}^{-h}(s, \nabla v)=s T_{e}^{-h} \nabla v+(1-s) \nabla v$. Now we take the sum of the inequalities (14) and (15). Dividing by $h$ and noting that

$$
\frac{1}{h}\left(D_{e}^{h} f-D_{e}^{-h} f\right)=\frac{1}{h^{2}}\left(T_{e}^{h} f-2 f+T_{e}^{-h} f\right)=D_{e}^{h} D_{e}^{-h} f
$$

we get

$$
\begin{gathered}
\int_{\Omega_{3 R}} \eta^{2}\left|D_{e}^{h} \nabla v\right|^{2} \int_{0}^{1}(1-s)\left|\mu_{e}^{h}(s, \nabla v)\right|^{p-2} d s d x \\
+\int_{\Omega_{3 R}} \eta^{2}\left|D_{e}^{-h} \nabla v\right|^{2} \int_{0}^{1}(1-s)\left|\mu_{e}^{-h}(s, \nabla v)\right|^{p-2} d s d x \\
\quad \leq \int_{\Omega_{3 R}} \eta^{2} D_{e}^{h} D_{e}^{-h} \frac{|\nabla v|^{p}}{p} d x-\int_{\Omega_{3 R}} \eta^{2}|\nabla v|^{p-2}\left\langle\nabla v, D_{e}^{h} D_{e}^{-h} \nabla v\right\rangle d x .
\end{gathered}
$$

Taking Lemma 3.1 into account (with $\alpha=1$ and $\beta=p-2$, implying $c_{1}=c_{1}(p)$ ) the assertion follows.

Now, we investigate the regularity of $u$ in a direction tangential to $\Gamma_{k_{0}}$ and prove the following result.

Lemma 3.3. Let the unit vector e be parallel to $\partial \Omega \cap B_{4 R}$. There is a positive constant $C=C(R, p)$ such that

$$
\begin{aligned}
& \sup _{0<h<\frac{R}{2}} \int_{\Omega_{R}}\left(\left|T_{e}^{h} \nabla u\right|^{2}+|\nabla u|^{2}\right)^{\frac{p-2}{2}}\left|D_{e}^{h} \nabla u\right|^{2} d x \\
&+\sup _{0<h<\frac{R}{2}} \int_{\Omega_{R}}\left(\left|T_{e}^{-h} \nabla u\right|^{2}+|\nabla u|^{2}\right)^{\frac{p-2}{2}}\left|D_{e}^{-h} \nabla u\right|^{2} d x \\
& \quad \leq C\left(\|\nabla u\|_{L^{p}\left(\Omega_{4 R}\right)}^{p}+\|f\|_{\left.W^{1, p^{\prime}\left(\Omega_{3 R}\right)}\right)}^{p^{\prime}}\right)
\end{aligned}
$$

Proof. Let $0<h<\frac{R}{2}$ and $\phi=\eta^{2} D_{e}^{h} D_{e}^{-h} u$, where $\eta$ is a cut-off function as in the previous lemma. Recall that $e$ is parallel to $\partial \Omega \cap B_{4 R}$. Thus, it follows that $\phi=0$ on $\partial \Omega$. Hence, the function $\phi$ is an admissible test function in equation (5). This yields

$$
\begin{aligned}
\int_{\Omega_{3 R}}|\nabla u|^{p-2} \partial_{i} u \eta^{2} \partial_{i} D_{e}^{h} D_{e}^{-h} u d x & \\
& =-\int_{\Omega_{3 R}}|\nabla u|^{p-2} \partial_{i} u \partial_{i} \eta^{2} D_{e}^{h} D_{e}^{-h} u d x+\int_{\Omega_{3 R}} f \eta^{2} D_{e}^{h} D_{e}^{-h} u d x .
\end{aligned}
$$


Utilizing Lemma 3.2 we obtain

$$
\begin{aligned}
c_{1} \int_{\Omega_{3 R}} \eta^{2} & \left(\left|T_{e}^{h} \nabla u\right|^{2}+|\nabla u|^{2}\right)^{\frac{p-2}{2}}\left|D_{e}^{h} \nabla u\right|^{2} d x \\
+c_{1} \int_{\Omega_{3 R}} \eta^{2} & \left(\left|T_{e}^{-h} \nabla u\right|^{2}+|\nabla u|^{2}\right)^{\frac{p-2}{2}}\left|D_{e}^{-h} \nabla u\right|^{2} d x \\
\leq & \frac{1}{p} \int_{\Omega_{3 R}} \eta^{2} D_{e}^{h} D_{e}^{-h}|\nabla u|^{p} d x+\int_{\Omega_{3 R}}|\nabla u|^{p-2} \partial_{i} u \partial_{i} \eta^{2} D_{e}^{h} D_{e}^{-h} u d x \\
& -\int_{\Omega_{3 R}} f \eta^{2} D_{e}^{h} D_{e}^{-h} u d x=: J_{1}+J_{2}+J_{3} .
\end{aligned}
$$

Now let us estimate the integrals $J_{1}, J_{2}$, and $J_{3}$ step by step. Notice that $\eta=0$ in $\Omega_{3 R} \backslash \Omega_{2 R}=\left(\Omega \cap B_{3 R}\right) \backslash\left(\Omega \cap B_{2 R}\right)$. Using the Leibniz rule for second order difference quotients

$$
\begin{aligned}
\eta^{2} D_{e}^{h} D_{e}^{-h}|\nabla u|^{p}= & D_{e}^{h} D_{e}^{-h}\left(\eta^{2}|\nabla u|^{p}\right)-T_{e}^{h}|\nabla u|^{p} D_{e}^{h} D_{e}^{-h} \eta^{2} \\
& -D_{e}^{-h} \eta^{2} D_{e}^{h}|\nabla u|^{p}-D_{e}^{-h} \eta^{2} D_{e}^{-h}|\nabla u|^{p}
\end{aligned}
$$

we find

$$
\begin{aligned}
J_{1}= & \frac{1}{p} \int_{\Omega_{3 R}} D_{e}^{h} D_{e}^{-h}\left(\eta^{2}|\nabla u|^{p}\right) d x-\frac{1}{p} \int_{\Omega_{3 R}} D_{e}^{h} D_{e}^{-h} \eta^{2} T_{e}^{h}|\nabla u|^{p} d x \\
& -\frac{1}{p} \int_{\Omega_{3 R}} D_{e}^{h}|\nabla u|^{p} D_{e}^{-h} \eta^{2} d x-\frac{1}{p} \int_{\Omega_{3 R}} D_{e}^{-h}|\nabla u|^{p} D_{e}^{-h} \eta^{2} d x \\
= & : J_{11}+\cdots+J_{14}
\end{aligned}
$$

In view of the facts that $e$ is parallel to $\partial \Omega \cap B_{4 R}$ and $\eta=0$ in $\Omega \backslash \Omega_{2 R}$ it follows

$$
\begin{aligned}
J_{11} & =\frac{1}{p h^{2}}\left(\int_{\Omega_{3 R}} T_{e}^{h}\left(\eta^{2}|\nabla u|^{p}\right) d x-2 \int_{\Omega_{3 R}} \eta^{2}|\nabla u|^{p} d x+\int_{\Omega_{3 R}} T_{e}^{-h}\left(\eta^{2}|\nabla u|^{p}\right) d x\right) \\
& =\frac{1}{p h^{2}}\left(\int_{\Omega_{3 R}+h e} \eta^{2}|\nabla u|^{p} d x-2 \int_{\Omega_{3 R}} \eta^{2}|\nabla u|^{p} d x+\int_{\Omega_{3 R}-h e} \eta^{2}|\nabla u|^{p} d x\right)
\end{aligned}
$$

and hence

$$
J_{11}=\frac{1-2+1}{p h^{2}} \int_{\Omega_{2 R}} \eta^{2}|\nabla u|^{p} d x=0 .
$$

Noting that $\left\|D_{e}^{h} D_{e}^{-h} \eta^{2}\right\|_{L^{\infty}\left(\Omega_{3 R}\right)} \leq C(R)$ we also get

$$
\left|J_{12}\right| \leq C(R, p) \int_{\Omega_{3 R}} T_{e}^{h}|\nabla u|^{p} d x=C(R, p) \int_{\Omega_{3 R}+h e}|\nabla u(y)|^{p} d y
$$


and hence

$$
\left|J_{12}\right| \leq C(R, p) \int_{\Omega_{\frac{7 R}{2}}}|\nabla u|^{p} d y \leq C(R, p)\|\nabla u\|_{L^{p}\left(\Omega_{4 R}\right)}^{p} .
$$

The Leibniz rule $g D_{e}^{h} f=D_{e}^{h}(f g)-T_{e}^{h} f D_{e}^{h} g$ yields

$$
J_{13}=-\frac{1}{p} \int_{\Omega_{3 R}} D_{e}^{h}\left(|\nabla u|^{p} D_{e}^{-h} \eta^{2}\right) d x+\frac{1}{p} \int_{\Omega_{3 R}} T_{e}^{h}|\nabla u|^{p} D_{e}^{h} D_{e}^{-h} \eta^{2} d x .
$$

The first integral on the right-hand side of (19) vanishes because of

$$
\begin{aligned}
\int_{\Omega_{3 R}} D_{e}^{h}\left(|\nabla u|^{p} D_{e}^{-h} \eta^{2}\right) d x & =\frac{1}{h} \int_{\left(\Omega_{3 R}+h e\right) \backslash \Omega_{3 R}}|\nabla u|^{p} D_{e}^{-h} \eta^{2} d x \\
& -\frac{1}{h} \int_{\Omega_{3 R} \backslash\left(\Omega_{3 R}+h e\right)}|\nabla u|^{p} D_{e}^{-h} \eta^{2} d x
\end{aligned}
$$

and the fact that $\eta=0$ in $\Omega \backslash \Omega_{2 R}$. Thus, we deduce

$$
\left|J_{13}\right| \leq C(R, p)\|\nabla u\|_{L^{p}\left(\Omega_{4 R}\right)}^{p} .
$$

Similarly, using the Leibniz rule $g D_{e}^{-h} f=D_{e}^{-h}(f g)-T_{e}^{-h} f D_{e}^{-h} g$ we find

$$
J_{14}=-\frac{1}{p} \int_{\Omega_{3 R}} D_{e}^{-h}\left(|\nabla u|^{p} D_{e}^{-h} \eta^{2}\right) d x+\frac{1}{p} \int_{\Omega_{3 R}} T_{e}^{-h}|\nabla u|^{p} D_{e}^{-h} D_{e}^{-h} \eta^{2} d x
$$

from which

$$
\left|J_{14}\right| \leq C(R, p)\|\nabla u\|_{L^{p}\left(\Omega_{4 R}\right)}^{p}
$$

follows. Next, utilizing again the Leibniz rule we have

$$
\begin{aligned}
J_{2}= & \int_{\Omega_{3 R}} D_{e}^{h}\left(|\nabla u|^{p-2} \partial_{i} u \partial_{i} \eta^{2} D_{e}^{-h} u\right) d x \\
& -\int_{\Omega_{3 R}} D_{e}^{h}\left(|\nabla u|^{p-2} \partial_{i} u \partial_{i} \eta^{2}\right) T_{e}^{h} D_{e}^{-h} u d x .
\end{aligned}
$$

Arguing as in (20) we see that the first integral on the right-hand side of (21) vanishes. For the second integral we apply once more the Leibniz rule to get

$$
\begin{aligned}
J_{2} & =-\int_{\Omega_{3 R}} D_{e}^{h}\left(|\nabla u|^{p-2} \partial_{i} u \partial_{i} \eta^{2}\right) D_{e}^{h} u d x \\
& =-\int_{\Omega_{3 R}}\left\{D_{e}^{h}\left(|\nabla u|^{p-2} \partial_{i} u\right) \partial_{i} \eta^{2}+T_{e}^{h}\left(|\nabla u|^{p-2} \partial_{i} u\right) D_{e}^{h} \partial_{i} \eta^{2}\right\} D_{e}^{h} u d x \\
& :=J_{21}+J_{22} .
\end{aligned}
$$


Recalling that $\operatorname{supp} \eta=\overline{B_{2 R}}$ the Hölder and Young inequalities yield

$$
\begin{aligned}
\left|J_{22}\right| & \leq C(\eta)\left(\int_{\Omega_{3 R}}\left|T_{e}^{h} \nabla u\right|^{p} d x\right)^{1-\frac{1}{p}}\left(\int_{\Omega_{3 R}}\left|D_{e}^{h} u\right|^{p} d x\right)^{\frac{1}{p}} \\
& \leq C(R, p)\|\nabla u\|_{L^{p}\left(\Omega_{4 R}\right)}^{p} .
\end{aligned}
$$

For estimating $J_{21}$ we use

$$
\begin{aligned}
\left|D_{e}^{h}\left(|\nabla u|^{p-2} \nabla u\right)\right|= & \left.\left|\int_{0}^{1}\right| \mu_{e}^{h}(s, \nabla u)\right|^{p-2} d s D_{e}^{h} \nabla u \\
& +(p-2) \int_{0}^{1}\left|\mu_{e}^{h}(s, \nabla u)\right|^{p-4}\left\langle\mu_{e}^{h}(s, \nabla u), D_{e}^{h} \nabla u\right\rangle \mu_{e}^{h}(s, \nabla u) d s \mid \\
& \leq(p-1) \int_{0}^{1}\left|\mu_{e}^{h}(s, \nabla u)\right|^{p-2} d s\left|D_{e}^{h} \nabla u\right| .
\end{aligned}
$$

Utilizing Hölder's and Young's inequalities, the properties of the cut-off function $\eta$, and Lemma 3.1 (with $\alpha=0, \beta=p-2$ ) we find

$$
\begin{aligned}
\left|J_{21}\right| \leq & 2(p-1) \int_{\Omega_{3 R}} \int_{0}^{1}\left|\mu_{e}^{h}(s, \nabla u)\right|^{p-2} d s\left|D_{e}^{h} \nabla u\right| \eta|\nabla \eta|\left|D_{e}^{h} u\right| d x \\
\leq & 2(p-1)\left(\int_{\Omega_{3 R}} \eta^{2} \int_{0}^{1}\left|\mu_{e}^{h}(s, \nabla u)\right|^{p-2} d s\left|D_{e}^{h} \nabla u\right|^{2} d x\right)^{\frac{1}{2}} \\
& \times\left(\int_{\Omega_{3 R}}|\nabla \eta|^{2} \int_{0}^{1}\left|\mu_{e}^{h}(s, \nabla u)\right|^{p-2} d s\left|D_{e}^{h} u\right|^{2} d x\right)^{\frac{1}{2}} \\
\leq & \frac{c_{1}}{4} \int_{\Omega_{3 R}} \eta^{2}\left(\left|T_{e}^{h} \nabla u\right|^{2}+|\nabla u|^{2}\right)^{\frac{p-2}{2}}\left|D_{e}^{h} \nabla u\right|^{2} d x \\
& +C(R, p) \int_{\Omega_{3 R}}\left(\left|T_{e}^{h} \nabla u\right|^{2}+|\nabla u|^{2}\right)^{\frac{p-2}{2}}\left|D_{e}^{h} u\right|^{2} d x \\
\leq & \frac{c_{1}}{4} \int_{\Omega_{3 R}} \eta^{2}\left(\left|T_{e}^{h} \nabla u\right|^{2}+|\nabla u|^{2}\right)^{\frac{p-2}{2}}\left|D_{e}^{h} \nabla u\right|^{2} d x+C(R, p)\|\nabla u\|_{L^{p}\left(\Omega_{4 R}\right)}^{p}
\end{aligned}
$$

Finally, noting that $\int_{\Omega_{3 R}} D_{e}^{h}\left(f \eta^{2} D_{e}^{-h} u\right) d x=0$ we get

$$
\begin{aligned}
J_{3} & =-\int_{\Omega_{3 R}} f \eta^{2} D_{e}^{h} D_{e}^{-h} u d x \\
& =-\int_{\Omega_{3 R}} D_{e}^{h}\left(f \eta^{2} D_{e}^{-h} u\right) d x+\int_{\Omega_{3 R}} D_{e}^{h}\left(f \eta^{2}\right) D_{e}^{h} u d x \\
& =\int_{\Omega_{3 R}} D_{e}^{h} u\left(D_{e}^{h} f \eta^{2}+T_{e}^{h} f D_{e}^{h} \eta^{2}\right) d x
\end{aligned}
$$


Therefore we have

$$
\left|J_{3}\right| \leq C(R, p)\left(\|\nabla u\|_{L^{p}\left(\Omega_{3 R}\right)}^{p}+\|f\|_{W^{1, p^{\prime}}\left(\Omega_{3 R}\right)}^{p^{\prime}}\right) .
$$

Collecting the results we conclude that

$$
\begin{aligned}
& \int_{\Omega_{R}}\left(\left|T_{e}^{h} \nabla u\right|^{2}+|\nabla u|^{2}\right)^{\frac{p-2}{2}}\left|D_{e}^{h} \nabla u\right|^{2} d x \\
& +\int_{\Omega_{R}}\left(\left|T_{e}^{-h} \nabla u\right|^{2}+|\nabla u|^{2}\right)^{\frac{p-2}{2}}\left|D_{e}^{-h} \nabla u\right|^{2} d x \\
& \leq C(R, p)\left(\|\nabla u\|_{L^{p}\left(\Omega_{4 R}\right)}^{p}+\|f\|_{W^{1, p^{\prime}\left(\Omega_{3 R}\right)}}^{p^{\prime}}\right)
\end{aligned}
$$

and the assertion of the lemma follows.

Next, let us study the regularity of $u$ in a direction normal to $\Gamma_{k_{0}}$.

Lemma 3.4. Let e be the inner unit normal of $\partial \Omega$. There is a positive constant $C=C(R, p)$ such that

$$
\begin{aligned}
\sup _{0<h<\frac{R}{2}} \int_{\Omega_{R}}\left(\left|T_{e}^{h} \nabla u\right|^{2}+|\nabla u|^{2}\right)^{\frac{p-2}{2}}\left|D_{e}^{h} \nabla u\right|^{2} d x & \\
+\sup _{0<h<\frac{R}{2}} \int_{\Omega_{R}}\left(\left|T_{e}^{-h} \nabla u\right|^{2}\right. & \left.+|\nabla u|^{2}\right)^{\frac{p-2}{2}}\left|D_{e}^{-h} \nabla u\right|^{2} d x \\
& \leq C\left(\|\nabla u\|_{L^{p}\left(\Omega_{4 R}\right)}^{p}+\|f\|_{W^{1, p^{\prime}\left(\Omega_{3 R}\right)}}^{p^{\prime}}\right)
\end{aligned}
$$

Proof. Let $0<h<\frac{R}{2}$. Further, let $z \in \partial \Omega \cap B_{3 R}$ and $z+\lambda e \in \Omega_{4 R}$. We extend the functions $u$ and $f$ onto $B_{4 R}$ by setting

$$
u(z-\lambda e):=-u(z+\lambda e) \text { and } \quad f(z-\lambda e):=f(z+\lambda e) .
$$

We define the set

$$
\Omega_{h}=\left\{y \in \Omega_{3 R}: y \neq x+h e, x \in \Omega_{3 R}\right\} \text {. }
$$

Furthermore, let $\Omega_{-h}$ be the reflection of $\Omega_{h}$ with respect to the hyperplane containing $\partial \Omega \cap B_{4 R}$.

Without loss of generality we may assume that $e=e_{n}$ where $e_{n}$ is the $n$-th unit vector of $\mathbb{R}^{n}$ (for, a coordinate transformation does not change the structure of the equation). Let $\eta$ be a cut-off function as in Lemma 3.2. We test the equation (1) by $\phi=\eta^{2} D_{e}^{h} D_{e}^{-h} u$. Let us verify that $\phi$ is an admissible test 
function. For $x \in \partial \Omega \cap \partial \Omega_{2 R}$ we have $u(x+h e)=-u(x-h e)$ and $u(x)=0$. Thus, $\phi=0$ on $\partial \Omega$ holds. We obtain

$$
\begin{aligned}
\int_{\Omega_{3 R}}|\nabla u|^{p-2} \partial_{i} u \eta^{2} \partial_{i} D_{e}^{h} D_{e}^{-h} u d x & \\
& =-\int_{\Omega_{3 R}}|\nabla u|^{p-2} \partial_{i} u \partial_{i} \eta^{2} D_{e}^{h} D_{e}^{-h} u d x+\int_{\Omega_{3 R}} f \eta^{2} D_{e}^{h} D_{e}^{-h} u d x .
\end{aligned}
$$

In view of Lemma 3.2 we get

$$
\begin{aligned}
& c_{1} \int_{\Omega_{3 R}} \eta^{2}\left(\left|T_{e}^{h} \nabla u\right|^{2}+|\nabla u|^{2}\right)^{\frac{p-2}{2}}\left|D_{e}^{h} \nabla u\right|^{2} d x \\
&+c_{1} \int_{\Omega_{3 R}} \eta^{2}\left(\left|T_{e}^{-h} \nabla u\right|^{2}+|\nabla u|^{2}\right)^{\frac{p-2}{2}}\left|D_{e}^{-h} \nabla u\right|^{2} d x \\
& \leq \frac{1}{p} \int_{\Omega_{3 R}} \eta^{2} D_{e}^{h} D_{e}^{-h}|\nabla u|^{p} d x \\
& \quad+\int_{\Omega_{3 R}}|\nabla u|^{p-2} \partial_{i} u \partial_{i} \eta^{2} D_{e}^{h} D_{e}^{-h} u d x-\int_{\Omega_{3 R}} f \eta^{2} D_{e}^{h} D_{e}^{-h} u d x \\
&=: J_{4}+J_{5}+J_{6} .
\end{aligned}
$$

Proceeding as in the proof of Lemma 3.3 we conclude that

$$
\begin{aligned}
J_{4}= & \frac{1}{p} \int_{\Omega_{3 R}} D_{e}^{h} D_{e}^{-h}\left(\eta^{2}|\nabla u|^{p}\right) d x-\frac{1}{p} \int_{\Omega_{3 R}} D_{e}^{h} D_{e}^{-h} \eta^{2} T_{e}^{h}|\nabla u|^{p} d x \\
& -\frac{1}{p} \int_{\Omega_{3 R}} D_{e}^{h}|\nabla u|^{p} D_{e}^{-h} \eta^{2} d x-\frac{1}{p} \int_{\Omega_{3 R}} D_{e}^{-h}|\nabla u|^{p} D_{e}^{-h} \eta^{2} d x \\
= & : J_{41}+\cdots+J_{44} .
\end{aligned}
$$

Now let us estimate the integrals $J_{41}, J_{43}$, and $J_{44}$. Notice that

$$
\begin{gathered}
\int_{\Omega_{3 R}} T_{e}^{h}\left(\eta^{2}|\nabla u|^{p}\right) d x-\int_{\Omega_{3 R}} \eta^{2}|\nabla u|^{p} d x=-\int_{\Omega_{h}} \eta^{2}|\nabla u|^{p} d x \\
\int_{\Omega_{3 R}} T_{e}^{-h}\left(\eta^{2}|\nabla u|^{p}\right) d x-\int_{\Omega_{3 R}} \eta^{2}|\nabla u|^{p} d x=\int_{\Omega_{-h}} \eta^{2}|\nabla u|^{p} d x .
\end{gathered}
$$

Thus, we have

$$
J_{41}=-\frac{1}{p h^{2}} \int_{\Omega_{h}} \eta^{2}|\nabla u|^{p} d x+\frac{1}{p h^{2}} \int_{\Omega_{-h}} \eta^{2}|\nabla u|^{p} d x=0 .
$$

Further, applying the Leibniz rule $D_{e}^{h} f D_{e}^{-h} g=D_{e}^{h}\left(f D_{e}^{-h} g\right)-T_{e}^{h} f D_{e}^{h} D_{e}^{-h} g$ we obtain

$$
J_{43}=-\frac{1}{p} \int_{\Omega_{3 R}} D_{e}^{h}\left(|\nabla u|^{p} D_{e}^{-h} \eta^{2}\right) d x+\frac{1}{p} \int_{\Omega_{3 R}} T_{e}^{h}|\nabla u|^{p} D_{e}^{h} D_{e}^{-h} \eta^{2} d x .
$$


Similarly, the identity $D_{e}^{-h} f D_{e}^{-h} g=D_{e}^{-h}\left(f D_{e}^{h} g\right)-f D_{e}^{h} D_{e}^{-h} g$ implies that

$$
J_{44}=-\frac{1}{p} \int_{\Omega_{3 R}} D_{e}^{-h}\left(|\nabla u|^{p} D_{e}^{h} \eta^{2}\right) d x+\frac{1}{p} \int_{\Omega_{3 R}}|\nabla u|^{p} D_{e}^{h} D_{e}^{-h} \eta^{2} d x .
$$

It is easy to see that

$$
\begin{aligned}
& -\frac{1}{p} \int_{\Omega_{3 R}} D_{e}^{h}\left(|\nabla u|^{p} D_{e}^{-h} \eta^{2}\right) d x-\frac{1}{p} \int_{\Omega_{3 R}} D_{e}^{-h}\left(|\nabla u|^{p} D_{e}^{h} \eta^{2}\right) d x \\
& \quad=\frac{1}{p h^{2}} \int_{\Omega_{h}}|\nabla u|^{p}\left(\eta^{2}-T_{e}^{-h} \eta^{2}\right) d x+\frac{1}{p h^{2}} \int_{\Omega_{-h}}|\nabla u|^{p}\left(T_{e}^{h} \eta^{2}-\eta^{2}\right) d x=0 .
\end{aligned}
$$

Furthermore, arguing as above (see (18)) we estimate the integral $J_{42}$. Altogether, we conclude that

$$
\left|J_{4}\right| \leq C(R, p)\|\nabla u\|_{L^{p}\left(\Omega_{4 R}\right)}^{p} .
$$

Now let us consider the integral $J_{5}$. Recall that $e=e_{n}$. Thus, the functions $|\nabla u|^{p-2} \partial_{i} u \partial_{i} \eta^{2} D_{e}^{h} D_{e}^{-h} u, 1 \leq i \leq n$, are even with respect to the hyperplane containing $\partial \Omega \cap B_{4 R}$, because, by the definitions of the extensions,

i) $|\nabla u|^{p-2}$ is even,

ii) $\partial_{i} u$ is odd for $i \neq n$ and even for $i=n$,

iii) $\partial_{i} \eta^{2}=2 \eta \partial_{i} \eta$ is even for $i \neq n$ and odd for $i=n$ (using a suitable radial-symmetric cut-off function),

iv) $D_{e}^{h} D_{e}^{-h} u$ is odd.

This yields

$$
J_{5}=\int_{\Omega_{3 R}}|\nabla u|^{p-2} \partial_{i} u \partial_{i} \eta^{2} D_{e}^{h} D_{e}^{-h} u d x=\frac{1}{2} \int_{B_{3 R}}|\nabla u|^{p-2} \partial_{i} u \partial_{i} \eta^{2} D_{e}^{h} D_{e}^{-h} u d x .
$$

Now we have an integral over $B_{3 R}$ and the support of the integrand is $B_{2 R}$. The identity $D_{e}^{h}(f g)=D_{e}^{h} f g+T_{e}^{h} f D_{e}^{h} g$ entails

$$
\begin{aligned}
J_{5} & =-\frac{1}{2} \int_{B_{3 R}} D_{e}^{h}\left(|\nabla u|^{p-2} \partial_{i} u \partial_{i} \eta^{2}\right) D_{e}^{h} u d x \\
& =-\frac{1}{2} \int_{B_{3 R}}\left\{D_{e}^{h}\left(|\nabla u|^{p-2} \partial_{i} u\right) \partial_{i} \eta^{2}+T_{e}^{h}\left(|\nabla u|^{p-2} \partial_{i} u\right) D_{e}^{h} \partial_{i} \eta^{2}\right\} D_{e}^{h} u d x .
\end{aligned}
$$

Proceeding from here as in estimating the integral $J_{2}$ in the proof of Lemma 3.3 we obtain

$$
\begin{aligned}
\left|J_{5}\right| \leq & \frac{c_{1}}{4} \int_{B_{3 R}} \eta^{2}\left(\left|T_{e}^{h} \nabla u\right|^{2}+|\nabla u|^{2}\right)^{\frac{p-2}{2}}\left|D_{e}^{h} \nabla u\right|^{2} d x+C(R, p)\|\nabla u\|_{L^{p}\left(\Omega_{4 R}\right)}^{p} \\
= & \frac{c_{1}}{4} \int_{\Omega_{3 R}} \eta^{2}\left(\left|T_{e}^{h} \nabla u\right|^{2}+|\nabla u|^{2}\right)^{\frac{p-2}{2}}\left|D_{e}^{h} \nabla u\right|^{2} d x \\
& +\frac{c_{1}}{4} \int_{\Omega_{3 R}} \eta^{2}\left(\left|T_{e}^{-h} \nabla u\right|^{2}+|\nabla u|^{2}\right)^{\frac{p-2}{2}}\left|D_{e}^{-h} \nabla u\right|^{2} d x+C(R, p)\|\nabla u\|_{L^{p}\left(\Omega_{4 R}\right)}^{p} .
\end{aligned}
$$


Finally, let us estimate $J_{6}$. Let us note that the integrand $f \eta^{2} D_{e}^{h} D_{e}^{-h} u$ is even with respect to the hyperplane containing $\partial \Omega \cap B_{4 R}$ because all of its factors are even (this follows for $f$ and $D_{e}^{h} D_{e}^{-h} u$ by the definitions of the extensions, and for $\eta$ we use a radial-symmetric cut-off function). We find

$$
\begin{aligned}
J_{6} & =-\int_{\Omega_{3 R}} f \eta^{2} D_{e}^{h} D_{e}^{-h} u d x \\
& =-\frac{1}{2} \int_{B_{3 R}} f \eta^{2} D_{e}^{h} D_{e}^{-h} u d x \\
& =-\frac{1}{2} \int_{B_{3 R}} D_{e}^{h}\left(f \eta^{2} D_{e}^{-h} u\right) d x+\frac{1}{2} \int_{B_{3 R}} D_{e}^{h}\left(f \eta^{2}\right) D_{e}^{h} u d x
\end{aligned}
$$

Noting that the first integral on the right-hand side vanishes we conclude that

$$
\left|J_{6}\right| \leq C(R, p)\left(\|\nabla u\|_{L^{p}\left(\Omega_{3 R}\right)}^{p}+\|f\|_{W^{1, p^{\prime}}\left(\Omega_{3 R}\right)}^{p^{\prime}}\right) .
$$

Thus, the assertion follows.

\section{Local regularity up to a non-smooth boundary}

In this section we are concerned with the case that $\partial \Omega \cap B_{4 R}(P)$ is not contained in a hyperplane. Thus, there is an index set $\Lambda$ such that $\Gamma_{k} \cap B_{4 R}(P) \neq \emptyset$ for all $k \in \Lambda, \partial \Omega \cap B_{4 R}(P)=\bigcup_{k \in \Lambda} \overline{\Gamma_{k}} \cap B_{4 R}(P)$, and $|\Lambda| \geq 2$. In this section we treat the case that $P \in \bigcap_{k \in \Lambda} \overline{\Gamma_{k}}$. Further, we suppose that $k_{0} \in \Lambda$ is fixed and $e \in \mathbb{R}^{d}$ is a unit vector parallel to $\left(\partial \Omega \cap B_{4 R}\right) \backslash \Gamma_{k_{0}}$ satisfying

$$
z+s e \in \bar{\Omega} \text { for } z \in \partial \Omega \cap B_{3 R}, s>0, \text { and } z+s e \in B_{4 R} .
$$

Let us note that $\partial \Gamma_{k_{1}} \cap \ldots \cap \partial \Gamma_{k_{j}}=\emptyset$, if $j>n$ and $k_{1}<\ldots<k_{j}$. Thus, there is at least one unit vector $e$ fulfilling (23). Now we prove

Lemma 4.1. Let $e \in \mathbb{R}^{n}$ be parallel to $\left(\partial \Omega \cap B_{4 R}\right) \backslash \Gamma_{k_{0}}$, across $\Gamma_{k_{0}}$, and let it satisfy (23). Then there is a positive constant $C=C(R, p)$ such that

$$
\begin{aligned}
& \sup _{0<h<\frac{R}{2}} \int_{\Omega_{R}}\left(\left|T_{*}^{h} \nabla u\right|^{2}+|\nabla u|^{2}\right)^{\frac{p-2}{2}}\left|D_{*}^{h} \nabla u\right|^{2} d x \\
&+\sup _{0<h<\frac{R}{2}} \int_{\Omega_{R}}\left(\left|T_{*}^{-h} \nabla u\right|^{2}+|\nabla u|^{2}\right)^{\frac{p-2}{2}}\left|D_{*}^{-h} \nabla u\right|^{2} d x \\
& \leq C\left(\|\nabla u\|_{L^{p}\left(\Omega_{4 R}\right)}^{p}+\|f\|_{W^{1, p^{\prime}\left(\Omega_{3 R}\right)}}^{p^{\prime}}\right) .
\end{aligned}
$$

The notation $T_{*}^{h}$ and $D_{*}^{ \pm h}$ will be defined immediately in the following proof. 
Proof. Let $0<h<\frac{R}{2}$. Further, let us note that $\Omega_{4 R}$ is convex. This fact will be used in the following reflection argument. Let $\sigma\left(\Omega_{4 R}\right)$ be the reflection of $\Omega_{4 R}$ with respect to the hyperplane containing $\Gamma_{k_{0}}$. Moreover, let $\sigma(e)$ be the reflection of $e$ and $\sigma(\partial \Omega)$ the reflection of $\partial \Omega$.

Let $z \in \Gamma_{k_{0}} \cap B_{3 R}$ and $z+\lambda e \in \Omega_{4 R}$. We extend $u$ and $f$ onto $\sigma\left(\Omega_{4 R}\right)$ by setting

$$
u(z+\lambda \sigma(e)):=-u(z+\lambda e), \quad f(z+\lambda \sigma(e)):=f(z+\lambda e) .
$$

Furthermore, let $z \in \Gamma_{k_{0}} \cap B_{3 R}$ be fixed. We define

$$
\psi(s)= \begin{cases}z+s e & \text { for } s \geq 0 \\ z-s \sigma(e) & \text { for } s<0\end{cases}
$$

Let $x \in \Omega_{3 R}$ be such that $x=z+s_{0} e$ for some $s_{0}>0$. Then we have $x=\psi\left(s_{0}\right)$. We now define

$$
\begin{aligned}
T_{*}^{h} f(x) & \equiv T_{*}^{h} f\left(\psi\left(s_{0}\right)\right):=f\left(\psi\left(s_{0}+h\right)\right) \\
T_{*}^{-h} f(x) & \equiv T_{*}^{-h} f\left(\psi\left(s_{0}\right)\right):=f\left(\psi\left(s_{0}-h\right)\right)
\end{aligned}
$$

and

$$
D_{*}^{h} f(x):=\frac{T_{*}^{h} f(x)-f(x)}{h}, \quad D_{*}^{-h} f(x):=\frac{f(x)-T_{*}^{-h} f(x)}{h} .
$$

Now, we follow the proof of Lemma 3.4. We use the test function $\phi=$ $\eta^{2} D_{*}^{h} D_{*}^{-h} u$ in equation (5). This is an admissible test function. On the one hand, $e$ is parallel to $\left(\partial \Omega \cap B_{4 R}\right) \backslash \Gamma_{k_{0}}$. Hence, $x \in\left(\partial \Omega \cap B_{3 R}\right) \backslash \Gamma_{k_{0}}$ implies that $T_{*}^{ \pm h} x \in(\partial \Omega \cup \sigma(\partial \Omega)) \cap B_{4 R} \backslash \Gamma_{k_{0}}$. On the other hand, for $z \in \Gamma_{k_{0}} \cap B_{3 R}$ it holds that $T_{*}^{-h} u(z)=-T_{*}^{h} u(z)$ and $u(z)=0$; thus, $T_{*}^{h} u(z)-2 u(z)+T_{*}^{-h} u(z)=0$. Hence, testing the equation and utilizing an analogue of Lemma 3.2 by using $T_{*}^{h}, T_{*}^{-h}$ and $D_{*}^{h}, D_{*}^{-h}$ instead of $T_{e}^{h}, T_{e}^{-h}$ and $D_{e}^{h}, D_{e}^{-h}$ which one easily checks to be true by following the proof of Lemma 3.2, we find

$$
\begin{aligned}
& c_{1} \int_{\Omega_{3 R}} \eta^{2}\left(\left|T_{*}^{h} \nabla u\right|^{2}+|\nabla u|^{2}\right)^{\frac{p-2}{2}}\left|D_{*}^{h} \nabla u\right|^{2} d x \\
& +c_{1} \int_{\Omega_{3 R}} \eta^{2}\left(\left|T_{*}^{-h} \nabla u\right|^{2}+|\nabla u|^{2}\right)^{\frac{p-2}{2}}\left|D_{*}^{-h} \nabla u\right|^{2} d x \\
& \leq \frac{1}{p} \int_{\Omega_{3 R}} \eta^{2} D_{*}^{h} D_{*}^{-h}|\nabla u|^{p} d x \\
& \quad+\int_{\Omega_{3 R}}|\nabla u|^{p-2} \partial_{i} u \partial_{i} \eta^{2} D_{*}^{h} D_{*}^{-h} u d x-\int_{\Omega_{3 R}} f \eta^{2} D_{*}^{h} D_{*}^{-h} u d x .
\end{aligned}
$$

Proceeding as in the proof of Lemma 3.4 and estimating the integrals on the right-hand side in a similar fashion as before the assertion follows. 


\section{Proof of the main results}

In this section we give the proofs of the Theorems 2.1 and 2.2. First of all, we prove the following proposition under the assumption that the right-hand side $f$ of the equation is sufficiently smooth, i.e., $f \in W^{1, p^{\prime}}(\Omega)$.

Proposition 5.1. Let $p>2$ and $f \in W^{1, p^{\prime}}(\Omega)$. Then the weak solution $u$ of (5) satisfies

$$
|\nabla u|^{\frac{p-2}{2}} \nabla u \in W^{1,2}\left(\Omega ; \mathbb{R}^{n}\right) .
$$

Proof. We cover $\Omega$ by a finite number of appropriate balls $B_{R_{i}}\left(P_{i}\right), i=1,2, \ldots$, such that either $B_{R_{i}}\left(P_{i}\right) \subset \subset \Omega$ or $P_{i} \in \partial \Omega$. First, let us consider the case that $B_{R_{i}}\left(P_{i}\right) \subset \subset \Omega$. Let $e \in \mathbb{R}^{n}$ be a unit vector. Following the proof of Lemma 3.3 we get a constant $C\left(R_{i}\right)$ depending only on $R_{i}$ and the data but not on $e$ such that

$$
\sup _{0<h<\frac{R_{i}}{2}} \int_{\Omega_{R_{i}}}\left(\left|T_{e}^{h} \nabla u\right|^{2}+|\nabla u|^{2}\right)^{\frac{p-2}{2}}\left|D_{e}^{h} \nabla u\right|^{2} d x \leq C\left(R_{i}\right) .
$$

Next, in the case that $P_{i} \in \partial \Omega$ we assume that $P_{i} \in \bigcap_{k \in \Lambda_{i}} \overline{\Gamma_{k}}$, where $\Lambda_{i}$ is an index set such that $\Gamma_{k} \cap B_{R_{i}}\left(P_{i}\right) \neq \emptyset$ for all $k \in \Lambda_{i}$ and $\partial \Omega \cap B_{R_{i}}\left(P_{i}\right)=$ $\bigcup_{k \in \Lambda_{i}} \overline{\Gamma_{k}} \cap B_{R_{i}}\left(P_{i}\right)$. If $\partial \Omega \cap B_{R_{i}}\left(P_{i}\right)$ is smooth, there are $n-1$ unit vectors parallel and one normal to the boundary. More general, we may choose $n$ linearly independent unit vectors fulfilling the assumptions of either Lemma 3.3, 3.4, or 4.1. We deduce (26) for these unit vectors $e$.

Now let $F(a)=|a|^{\frac{p-2}{2}} a\left(a \in \mathbb{R}^{n}\right)$ and $F_{i}(a)=\frac{\partial}{\partial a_{i}} F(a)$. Utilizing the Taylor expansion and Lemma 3.1 we find

$$
\begin{aligned}
|F(a)-F(b)|^{2} & =\left|(a-b)_{i} \int_{0}^{1} F_{i}(t a+(1-t) b) d t\right|^{2} \\
& \leq c\left(|a|^{2}+|b|^{2}\right)^{\frac{p-2}{2}}|a-b|^{2} .
\end{aligned}
$$

Due to $(26)$ we conclude there is a constant $C\left(R_{i}\right)$ such that

$$
\sup _{0<h<\frac{R_{i}}{2}}\left\|D_{e}^{h} F(\nabla u)\right\|_{L^{2}\left(\Omega_{R_{i}}\right)}^{2} \leq C\left(R_{i}\right) .
$$

Hence, there is a constant $C_{0}$ depending only on the geometry of $\partial \Omega$ and the data such that

$$
\sup _{e \in \mathbb{R}^{n},|e|=1} \sup _{h>0}\left\|D_{e}^{h} F(\nabla u)\right\|_{L^{2}\left(\Omega^{h}\right)}^{2} \leq C_{0},
$$

where $\Omega^{h}=\{x \in \Omega: \operatorname{dist}(x, \partial \Omega) \geq h\}$. This implies that $\|\nabla F(\nabla u)\|_{L^{2}(\Omega)}^{2} \leq C_{0}$. Thus, the assertion follows. 
Remark. i) It is easy to see that

$$
\left|\partial_{e}\left(|\nabla u|^{\frac{p-2}{2}} \nabla u\right)\right|^{2} \equiv\left|\partial_{e} F(\nabla u)\right|^{2} \geq|\nabla u|^{p-2}\left|\partial_{e} \nabla u\right|^{2},
$$

where $\partial_{e}$ is the derivative in direction $e$. From Proposition 5.1 it follows that

$$
\int_{\Omega}|\nabla u|^{p-2}\left|\nabla^{2} u\right|^{2} d x \leq C
$$

ii) In (27) we have shown $\left.|| a\right|^{\frac{p-2}{2}} a-\left.|b|^{\frac{p-2}{2}} b\right|^{2} \leq c\left(|a|^{2}+|b|^{2}\right)^{\frac{p-2}{2}}|a-b|^{2}$. In the same manner it follows there is a constant $c^{\prime}>0$ such that

$$
\left.|| a\right|^{\frac{p-2}{2}} a-\left.|b|^{\frac{p-2}{2}} b\right|^{2} \geq c^{\prime}\left(|a|^{2}+|b|^{2}\right)^{\frac{p-2}{2}}|a-b|^{2} .
$$

Further, it is well-known (see $[2,3])$ that there is a constant $c^{\prime \prime}$ independent of $a$ and $b$ such that

$$
|a-b|^{p} \leq c^{\prime \prime}\left(|a|^{2}+|b|^{2}\right)^{\frac{p-2}{2}}|a-b|^{2} .
$$

Thus, putting $a=T_{e}^{h} \nabla u$ and $b=\nabla u$ we find constants $C$ and $C^{\prime}$ such that

$$
|u|_{\mathcal{N}^{1+\frac{2}{p}, p}(\Omega)}^{p} \leq C \sup _{e \in \mathbb{R}^{n},|e|=1} \sup _{h>0}\left\|D_{e}^{h}\left(|\nabla u|^{\frac{p-2}{2}} \nabla u\right)\right\|_{L^{2}\left(\Omega^{h}\right)}^{2},
$$

for

$$
\int_{\Omega_{h}} h^{-2}\left|T_{e}^{h} \nabla u-\nabla u\right|^{p} \leq c \int_{\Omega_{h}}\left|D_{e}^{h}\left(|\nabla u|^{\frac{p-2}{2}} \nabla u\right)\right|^{2}
$$

and

$$
\int_{\Omega^{h}}\left(\left|T_{e}^{h} \nabla u\right|^{2}+|\nabla u|^{2}\right)^{\frac{p-2}{2}}\left|D_{e}^{h} \nabla u\right|^{2} d x \geq C^{\prime}\left\|D_{e}^{h}\left(|\nabla u|^{\frac{p-2}{2}} \nabla u\right)\right\|_{L^{2}\left(\Omega^{h}\right)}^{2} .
$$

Proof of Theorem 2.2. Notice that $f \in W^{\frac{p-2}{p}, p^{\prime}}(\Omega)$. Let $\left(f^{k}\right)_{k \in \mathbb{N}}$ be a sequence of $W^{1, p^{\prime}}(\Omega)$-functions such that $f^{k} \rightarrow f$ in $W^{\frac{p-2}{p}, p^{\prime}}(\Omega)$. Then there is a sequence of functions $\left(u^{k}\right)_{k \in \mathbb{N}}$ solving in the weak sense

$$
-\operatorname{div}\left(\left|\nabla u^{k}\right|^{p-2} \nabla u^{k}\right)=f^{k} \quad \text { in } \Omega, \quad u^{k}=0 \quad \text { on } \partial \Omega .
$$

The proofs of (5.1) and (29) imply that

$$
\int_{\Omega}\left|\nabla\left(\left|\nabla u^{k}\right|^{\frac{p-2}{2}} \nabla u^{k}\right)\right|^{2} d x+\int_{\Omega}\left|\nabla u^{k}\right|^{p-2}\left|\nabla^{2} u^{k}\right|^{2} d x \leq C\left\|f^{k}\right\|_{W^{s, p^{\prime}}(\Omega)}^{p^{\prime}}
$$

for $s=1$ and a constant $C$ depending only on $p, n$, and $\partial \Omega$. Now let us show that this estimate holds for $s=\frac{p-2}{p}$, as well. Therefore, we have to modify the proofs 
of the Lemmas 3.3, 3.4, and 4.1, and to give an estimate of $\left|\int \eta^{2} D_{e}^{h} u^{k} D_{e}^{h} f^{k}\right|$ by using only the $W^{\frac{p-2}{p}, p^{\prime}}(\Omega)$-norm of $f^{k}$. Let $\varepsilon>0$. We deduce

$$
\begin{aligned}
\left|\int_{B_{3 R}} \eta^{2} D_{e}^{h} u^{k} D_{e}^{h} f^{k} d x\right| & \leq\left\|\eta^{2} D_{e}^{h} u^{k}\right\|_{W^{\frac{2}{p}, p}\left(B_{3 R}\right)}\left\|D_{e}^{h} f^{k}\right\|_{W^{-\frac{2}{p}, p^{\prime}}{ }_{\left(B_{3 R}\right)}} \\
& \leq \varepsilon\left\|u^{k}\right\|_{\mathcal{N}^{1+\frac{2}{p}, p}\left(B_{3 R}\right)}^{p}+C_{\varepsilon}\left\|f^{k}\right\|_{W^{\frac{p-2}{p}, p^{\prime}\left(B_{3 R}\right)}}^{p^{\prime}} .
\end{aligned}
$$

Using a covering argument, (32), (33), and choosing $\varepsilon>0$ sufficiently small we obtain estimate (34) for $s=\frac{p-2}{p}$. Further, due to the fact that $f^{k} \rightarrow f$ $(k \rightarrow \infty)$ in $W^{\frac{p-2}{p}, p^{\prime}}(\Omega)$ it follows that the integrals on the left-hand side of (34) are uniformly bounded. In view of (32) this implies that the $\mathcal{N}^{1+\frac{2}{p}, p}$-norms are uniformly bounded. Hence, there is a subsequence (again denoted by $\left(u^{k}\right)_{k}$ ) and a function $v \in W_{0}^{1, p}(\Omega)$ such that

$$
u^{k} \rightarrow v \quad(k \rightarrow \infty) \quad \text { strongly in } W_{0}^{1, p}(\Omega) .
$$

We deduce that there is a subsequence (denoted by $\left(u^{k}\right)_{k}$ ) such that $\nabla u^{k}(x) \rightarrow$ $\nabla v(x)$ a.e., thus,

$$
\left|\nabla u^{k}(x)\right|^{p-2} \nabla u^{k}(x) \rightarrow|\nabla v(x)|^{p-2} \nabla v(x) \quad \text { a.e. . }
$$

This implies that $\left|\nabla u^{k}\right|^{p-2} \nabla u^{k}$ converges weakly to $|\nabla v|^{p-2} \nabla v$ in $L^{p^{\prime}}(\Omega)$. Hence, $v$ is a weak solution of $-\Delta_{p} v=f$. We conclude that $v=u$.

Now let us investigate the regularity of $u$. Notice that $\nabla u^{k} \rightarrow \nabla u$ in $L^{p}(\Omega)$, and hence (cf. (27)) $\left|\nabla u^{k}\right|^{\frac{p-2}{2}} \nabla u^{k} \rightarrow|\nabla u|^{\frac{p-2}{2}} \nabla u$ in $L^{2}(\Omega)$. Thus we obtain by lower semicontinuity of the $L^{2}$-norm or by Fatou's lemma

$$
\left\|D_{e}^{h}\left(|\nabla u|^{\frac{p-2}{2}} \nabla u\right)\right\|_{L^{2}\left(\Omega^{h}\right)} \leq \liminf _{k \rightarrow \infty}\left\|D_{e}^{h}\left(\left|\nabla u^{k}\right|^{\frac{p-2}{2}} \nabla u^{k}\right)\right\|_{L^{2}\left(\Omega^{h}\right)} \leq C
$$

for $\left\|D_{e}^{h}\left|\nabla u^{k}\right|^{\frac{p}{2}}\right\|_{L^{2}\left(\Omega^{h}\right)}$ is uniformly bounded. Estimate (35) yields $|\nabla u|^{\frac{p-2}{2}} \nabla u \in$ $W^{1,2}(\Omega)$. Due to $(28)$ the assertion follows.

Proof of Theorem 2.1. Utilizing the estimates (32) and (35) it follows that $u \in \mathcal{N}^{1+\frac{2}{p}, p}(\Omega)$.

\section{References}

[1] Bensoussan, A. and J. Frehse: Asymptotic behaviour of Norton-Hoff's law in plasticity theory and $H^{1}$ regularity. In: Boundary Value Problems for Partial Differntial Equations and Applications (eds.: J. L. Lions and C. Baiocchi). Paris: Masson 1993, pp. $3-25$. 
[2] Chen, Y. Z. and E. DiBenedetto: Boundary estimates for solutions of nonlinear degenerate parabolic systems. J. Reine Angew. Math. 395 (1985), 102 - 131.

[3] DiBenedetto, E.: Degenerate Parabolic Equations. New York: Springer 1993.

[4] Ebmeyer, C.: Mixed boundary value problems for nonlinear elliptic systems in n-dimensional Lipschitzian domains. Zeit. Anal. Anwend. 18 (1999), 539 - 555.

[5] Ebmeyer, C.: Nonlinear elliptic problems with p-structure under mixed boundary value conditions in polyhedral domains. Adv. Diff. Equ. 6 (2001), 873- 895.

[6] Ebmeyer, C. and J. Frehse: Mixed boundary value problems for nonlinear elliptic equations in multidimensional non-smooth domains. Math. Nachr. 203 (1999), $47-74$.

[7] Ebmeyer, C. and WB. Liu: Quasi-norm interpolation error estimates for the piecewise linear finite element approximation of p-Laplacian problems. Numer. Math. 100 (2005), 233 - 258.

[8] Frehse, J., J. Málek and M. Steinhauer: On analysis of steady flows of fluids with shear dependent viscosity based on the Lipschitz truncation method. SIAM J. Math. Anal. 34 (2003), 1064 - 1083.

[9] Giusti, E.: Metodi Diretti nel Calcolo delle Variazioni (in Italian). Bologna: Unione Matematica Italiana 1994.

[10] Kufner, A., O. John and S. Fučik: Function Spaces. Prague: Academia 1977.

[11] Liu, WB.: Degenerate quasilinear elliptic equations arising from bimaterial problems in elastic-plastic mechanics. Nonlin. Anal. 35 (1999), 517 - 529.

[12] Liu, WB. and J. W. Barrett: A further remark on the regularity of the solutions of the p-Laplacian and its applications to their finite element approximation. Nonlin. Anal. 21 (1993), 379 - 387.

[13] Málek, J., J. Nečas, M. Rokyta and M. Růžička: Existence and Regularity of Solutions to Evolutional Partial Differential Equations. Applied Mathematics and Mathematical Computation, Vol. 13. London: Chapman and Hall 1996.

[14] Müller, M. and J. Nečas: Über die Regularität der schwachen Lösungen von Randwertaufgaben für quasilineare elliptische Differentialgleichungen höherer Ordnung (in German). Czech. Math. J. 25 (100) (1975), 227 - 239.

[15] Nečas, J.: Introduction to the Theory of Nonlinear Elliptic Equations. TeubnerTexte zur Mathematik, Bd. 52. Leipzig: Teubner 1983.

[16] Nikolskij, S. M.: Approximation of Functions of Several Variables and Imbedding Theorems. Berlin: Springer 1975.

[17] Tolksdorff, P.: Everywhere regularity for some quasi-linear systems with lack of ellipticity. Ann. Mat. Pura Appl. 134 (1983), 241 - 266.

Received 10.10.2003; in revised form 26.03.2004 